\title{
Mit Leben füllen
}

Man muss die Leitlinien kennen, um bewusst von ihnen abweichen zu können. Nur so lässt sich dann im individuellen Fall begründen, weshalb die aktuellen medizinischen Leitlinien verlassen wurden: Warum also wird zum Beispiel der schizophrene Patient nicht nur mit einem Antipsychotikum therapiert oder warum wird der depressive Patient nicht auch psychotherapeutisch behandelt?

In kaum einem Fachbereich der Medizin unterscheiden sich die theoretischen Diagnose- und

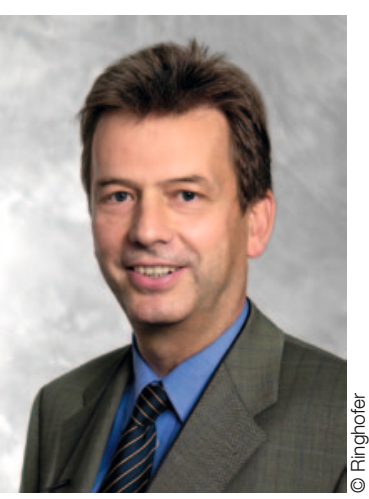

HERAUSGEBER / CHIEF EDITOR

Univ-.Prof. DDr. Peter Fischer Behandlungsrichtlinien und die tatsächlich durchgeführte Diagnostik und Therapie so deutlich wie in der Psychiatrie und medizinischen Psychotherapie. Diese Kluft zwischen Theorie und Praxis will die Psychopraxis seit jeher überbrücken. Ein Möglichkeit dazu bietet die Schilderung klinisch beobachteter Fälle. Dabei können der typische Fall, die diagnostische Überraschung, unerwartete Nebenwirkungen oder auch einzelne, sehr spezifische Fragen oder Beobachtungen, wert sein, mitgeteilt zu werden.

Ich bin überzeugt, dass sich alle Problemstellungen in Diagnose und Therapie neuropsychiatrischer Erkrankungen in Kasuistiken lebendig darstellen lassen. Deshalb möchte ich alle klinisch tätigen Kolleginnen und Kollegen der Fachgebiete Psychiatrie und medizinische Psychotherapie sowie Neurologie und verwandte Fachgebiete sehr herzlich einladen, solche Kasuistiken oder Fallserien für unsere Zeitschrift Psychopraxis zu verfassen.

Jeder Kasuistik sollte

einleitend vorangestellt sein, warum dieser Fall berichtenswert ist. Denn ohne theoretischen Hintergrund kann es keine neuen und guten Beob-

„Ich bin fest davon überzeugt, dass es ohne Vermutungen keine guten und neuartigen Beobachtungen gibt."

Charles R. Darwin

achtungen geben. Nach der

beliebig strukturierten Falldar-

stellung sollte in einem letzten Absatz kurz geschildert werden, was nun vor dem Hintergrund der State-of-the-Art-Medizin aus diesem Fall gelernt werden kann. Viele neue Richtlinien entwickelten sich ja erst aus der Beobachtung interessanter Einzelfälle. Die Fallschilderungen in der Psychopraxis sollen die bestehenden Richtlinien nicht ersetzen, sondern ergänzen und mit Leben füllen.

\section{Peter Fischer}

